DOI $10.35381 / \mathrm{cm} . v 6 \mathrm{i} 11.354$

\title{
La investigación formativa en el contexto de la universidad peruana
}

\section{Formative research in the context of the peruvian university}

\author{
Bryam Alejandro Colán-Hernández \\ 100061242@ucientifica.edu.pe \\ Universidad Científica del Sur, Lima \\ Perú \\ https://orcid.org/0000-0003-1976-9859
}

Recibido: 09 de abril de 2020

Revisado: 11 de mayo de 2020

Aprobado: 01 de julio de 2020

Publicado: 18 de julio de 2020

\section{RESUMEN}

El siguiente articulo hace una reflexión sobre los diferentes estereotipos de la investigación formativa que tienen las universidades peruanas y los relaciona con los paradigmas educativos contemporáneos que influyeron en la educación superior en los últimos 200 años. Se utiliza un enfoque cualitativo de investigación documental que nos permite una diferenciación conceptual clara entre los diferentes autores consultados a través de la interpretación hermenéutica. Finalmente, se concluye que la investigación formativa no debe partir de estereotipos sin base paradigmática y que los modelos educativos universitarios deben conectar con los paradigmas contemporáneos que tienen relación con las necesidades de aprendizajes y competencias profesionales para garantizar un capital profesional de calidad.

Descriptores: Enfoque científico; método de formación; organización de la investigación; programa de investigación. (Palabras tomadas del Tesauro UNESCO). 


\begin{abstract}
The following article makes a reflection on the different stereotypes of formative research that Peruvian universities have and relates them to the contemporary educational paradigms that influenced higher education in the last 200 years. A qualitative approach to documentary research is used that allows us a clear conceptual differentiation between the different authors consulted through hermeneutical interpretation. Finally, it is concluded that formative research should not start from stereotypes without a paradigmatic basis and that university educational models must connect with contemporary paradigms that are related to the needs of learning and professional skills to guarantee quality professional capital.
\end{abstract}

Descriptors: Scientific approach; training methods; organization of research; research programmes. (Words taken from the UNESCO Thesaurus).

\title{
INTRODUCCIÓN
}

La investigación formativa es un tema pendiente en la formación profesional universitaria a nivel mundial. Un problema medular, es la desvinculación de un propósito formativo por encima de un conjunto de cursos que conducen a la presentación de una tesis que asegurará un título profesional. Las investigaciones en torno a la investigación formativa están logrando visibilizar la importancia de la profundización del conocimiento y de la solución creativa de problemas a medida que se van adquiriendo competencias profesionales.

Sin embargo, su crecimiento no está asegurando la calidad de la investigación ni su capacidad de réplica, haciendo que la investigación sea una de las actividades más "odiadas" que se hacen en la universidad. Esto debido a los estereotipos de investigación centrados en la producción y no en la formación.

Por ello, el presente artículo pretende vincular el propósito de la investigación dentro de la formación, a través de la revisión de la literatura y de las experiencias que han corregido el problema y han tenido resultados sobresalientes desde los paradigmas educativos contemporáneos. Con ello, se busca repensar la estructura de la investigación formativa, su metodología, su funcionamiento, y su impacto sistematizando conceptos 
importantes que toda universidad debería considerar para la implementación y funcionamiento de una cultura investigativa.

Por lo tanto, este artículo analizará los estereotipos investigativos en la universidad peruana y los conceptos claves de la investigación formativa, identificando la problemática y los principales autores y aportes a la revisión de la literatura, así como los aportes paradigmáticos contemporáneos en educación, relacionados a la investigación formativa. Desde esta visión el objetivo del presente artículo es reflexionar reflexión sobre los diferentes estereotipos de la investigación formativa en el contento de la universidad peruana.

\section{METODOLOGÍA}

Para estudiar la investigación formativa, tomamos en cuenta el paradigma interpretativo, bajo el enfoque cualitativo, basado en la investigación documental, utilizando la hermenéutica para las comparaciones conceptuales. Para procesar la información, realizamos un análisis de contenido entre las definiciones conceptuales de los diferentes autores y la relación con los paradigmas contemporáneos.

\section{RESULTADOS}

Resulta una discusión muy sensible, delimitar los aspectos que abarcan la investigación formativa en la universidad (De la Ossa, et all, 2012). Hasta ahora, las universidades han tomado en cuenta, que la investigación formativa es lo que conduce a un grado formativo y lo que puede garantizar publicaciones o participación en eventos de la comunidad científica o intelectual. Esto en la actualidad, se viene traduciendo en un estancamiento irónico de publicaciones y proyectos de investigación conducentes a la obtención de grados y títulos. La asimilación de los conceptos científicos en la universidad ha devenido enteramente en normas y formatos que no brindan claridad respecto al sentido académico y profesional y han creado un estereotipo respecto a los paradigmas científicos vigentes y válidos y respecto al acceso meritocrático. 


\section{Bryam Alejandro Colán-Hernández}

Adicionalmente, debemos reflexionar sobre la relación entre la autonomía universitaria y la estandarización de la investigación, sobre todo de la investigación formativa (Restrepo, 2003). Por un lado, los estereotipos normalizan modelos de investigación universitarios centrados en la publicación de artículos, libros, monografías, póster y compilaciones (Calderón, 2015). Cuando pasan las evaluaciones de estandarización, la medición se hace desde este estereotipo y se invisibiliza la investigación formativa, ya que solo consideran los resultados, el impacto y la frecuencia de publicación.

Si el fin de la universidad es la creación de nuevo conocimiento (Tunnerman y De Souza, 2003), debería considerarse que esta creación es parte de un proceso metodológico y progresivo que vincula las necesidades del mercado con las habilidades investigativas que deben desarrollar los estudiantes universitarios y va más allá de una obligación productiva. Por lo tanto, dicho estereotipo es incompleto y deberíamos complementarlo desde la investigación formativa (Restrepo, 2003).

Por otro lado, la investigación formativa, sale del estereotipo de producción y entra al estereotipo didáctico. Dentro de las consideraciones didácticas de la investigación, la investigación formativa cobra un interés central dentro de los programas académicos universitarios. Los docentes deberán desarrollar creativamente las habilidades investigativas en sus estudiantes y asegurar que no se pierdan oportunidades de publicación o fondos, asegurando el paso de la investigación formativa a la investigación en sentido estricto. (Neihardt, 1997).

\section{DISCUSIÓN}

\section{Vinculaciones conceptuales entre la investigación formativa y los paradigmas educativos contemporáneos}

La investigación formativa es el conjunto de procesos curriculares y de gestión institucional que desarrolla la universidad para garantizar el logro de competencias investigativas, que formarán parte de las competencias con las que contará el egresado de la universidad, para enfrentar las exigencias del mercado laboral y la comunidad 
académica. Restrepo (2003), considera que la investigación formativa debe tomar en cuenta tres aspectos:

\section{La investigación exploratoria:}

“...búsqueda de necesidades, problemas, hipótesis y poblaciones relevantes para estructurar o refinar proyectos de investigación cuando éstos no tienen claros ni unas ni otros". (p.198)

Al respecto podemos encontrar relevante que la investigación exploratoria pueda vincular la problemática y el contexto donde está inmerso el estudiante y que le permiten formular preguntas relevantes, que posteriormente profundizará y responderá. Al respecto podemos vincular la investigación exploratoria con la búsqueda y organización de información desde el sentido común, necesaria en toda investigación exploratoria y presente en la teoría de la experiencia desarrollada por John Dewey (Ruiz, 2013). Por otro lado, siguiendo la teoría de juegos de Montessori (Lillard,2013), las habilidades investigativas adquiridas desde la primera infancia, se configuran desde la creatividad, el lenguaje y la imaginación, y representan las primeras iniciativas de investigación para el niño hasta alinearse con las normas y roles que perfeccionan dichas habilidades, por lo que el niño debe desarrollar una autoestima ${ }^{1}$ compatible con la autoeficacia y la autorregulación.

\section{La formación en y para la investigación:}

“...la función ínsita en esta acepción es la de aprender (formar en) la lógica y actividades propias de la investigación científica". (p.198)

Al respecto se puede entender que la investigación formativa debe desarrollar hábitos en el estudiante que le permitan comparar, contrastar, desechar o validar la información que

\footnotetext{
${ }^{1}$ Un estudio comparativo sobre autoestima entre niños de 9 a 12 años educados con el método tradicional y el método Montessori, reveló que los niños educados con el método Montessori tienen una autoestima altamente correlacionada con los niveles de autoeficacia y aprendizaje autorregulado. Véase. (Acevedo y Carrillo, 2010)
} 
ha encontrado, esta información deberá estar ajustada a los intereses del estudiante. Esto nos revela la necesidad de un diagnóstico sobre los intereses desde las habilidades investigativas que se han desarrollado desde la escuela, ya que, según Freinet, esto es fundamental para desarrollar interés por la ciencia y la producción científica (Santaela y Martinez, 2017).

Dichas actividades deben reflejar la coherencia con la intensión del estudiante, respecto a la relevancia de los datos que encuentra y que apoyan sus argumentos o de los que desechan las dudas que se puedan generar, producto de una constante revisión bibliográfica.

La investigación para la transformación en la acción o en la práctica:

“...investigación-acción o a aquella investigación realizada para aplicar sus hallazgos sobre la marcha, para afinar y mejorar los programas mientras están siendo desarrollados, para servir a los interesados como medio de reflexión y aprendizaje sobre sus programas y sus usuarios". (p.198)

Podemos entender, que luego del proceso exploratorio y de contrastación de la información, la investigación formativa debe transformar la actividad curricular y debe ajustarse a los propósitos de los programas de estudios, impactando en la profundidad y objetividad al momento de plantear alternativas o producir nuevo conocimiento. Así mismo, el redescubrimiento de resultados que pueden asegurar una práctica investigativa potencial, debería ser el norte del aprendizaje en la universidad, ya que no basta la enseñanza de la metodología y las habilidades, sino la práctica y el registro recurrente de las actividades investigativas. Siguiendo las reflexiones de Brunner, y considerando el fin de la universidad, el conocimiento que logramos adquirir, es el que redescubrimos (Guilar, 2009). 


\section{Integración e internacionalización de la investigación formativa.}

La investigación formativa en el contexto europeo, es un ejemplo de lo que América Latina puede lograr con la discusión sobre la apertura de sus servicios educativos universitarios. Europa ha tenido una tendencia integradora desde la declaración de Bolonia en 1999. El proyecto TUNING, acercó a las universidades entre sí para mantener una independencia de la gestión pública por país y avanzar al logro de competencias profesionales.

Larraín y González (2007) muestran que este logro de competencias profesionales, se dividen en generales y específicas. Considerando las generales, podemos encontrar la formación de las habilidades de investigación dentro de las competencias sistémicas. Éstas competencias se encargan de agrupar el conjunto de destrezas y habilidades que le permiten al estudiante una mejor comprensión, sensibilidad para el logro de un pensamiento sistémico. Así mismo se muestra en las competencias específicas, una preponderante importancia en la investigación cualitativa, así como en el manejo de herramientas y técnicas de procesamiento de los datos investigados.

\section{Repensando la investigación formativa para mejorar la enseñanza universitaria}

La investigación formativa es necesaria en el plan curricular y debe medirse desde las dimensiones curriculares que valoran el proceso investigativo hasta la publicación (Mendoza, 2016). Así mismo se debe evidenciar, que accesos a estas bases bibliográficas la universidad le brinda al estudiante y cómo la promueve, cómo se vinculan los trabajos que requieren redacción a las normas internacionales de publicación, como se interioriza la metodología de la investigación en la problemática que resuelve cada curso y como organizan e interpretan los datos para luego plantear soluciones (Moreno, 2011). Por otro lado, la investigación formativa es un proceso de generación de ideas, profundización y vinculación con el mundo real. se requiere una constante asociación de conceptos y métodos que determinan la modificación de conocimientos que son analizados y validados por los pares, Ruíz (2017). 
Metodologías como la Enseñanza Basado en Problemas deben proponerse en el sílabo para asegurar que la investigación formativa sea un proceso activo y dinámico, Ordoñez (2017). Este proceso heurístico, es fundamental para profundizar el conocimiento. Lo que no podemos negar es que, el proceso de investigación formativa debe estar en cada programa de cada carrera como pieza clave para sostener la empleabilidad y aplicar mejoras en las actividades profesionales, Corbella y Alonso (2019).

\section{CONCLUSIÓN:}

El estereotipo de la investigación científica centrado en la producción es incompleto, pues en el contexto de la universidad peruana se siguen estableciendo esquemas procedimentales, estructuras rígidas basada en una concepción paradigmática que no permite que emerja una investigación formativa desde las bases del colectivo docente que responda a las verdaderas necesidades de la formación en investigación.

El estereotipo de la investigación formativa centrado en la didáctica complementa el proceso de la investigación centrado en producción, es decir, la investigación formativa, se establece desde concepciones estructurales arraigadas en una visión paradigmática que no permite fomentar otros paradigmas dentro del proceso didáctico.

Las corrientes y teorías educativas contemporáneas pueden tomarse de base para desarrollar la propuesta de la investigación formativa y mejorar la producción científica de la universidad, basada en pensamiento flexible, sistémico, holístico y complementario cónsono con las realidades actuales a investigar.

\section{REFERENCIAS CONSULTADAS}

Calderón, R. (2015). La percepción de la ciencia, tecnología e innovación en estudiantes de nivel medio y superior de la zona metropolitana de Guadalajara, México. Revista iberoamericana parla la investigación y el desarrollo educativo. 6(11), pp. 1-24. Recuperado de: https://dialnet.unirioja.es/servlet/articulo?codigo=5280206 
Corbella, M. y Alonso, R. (2019). El vínculo universidad-empresa en las prácticas curriculares. Investigación e innovación. Contextos educativos, 23, pp. 7-9. doi: $10.18172 /$ con. 3742 . Recuperado de: https://dialnet.unirioja.es/descarga/articulo/6856438.pdf

De la Ossa, J., Pérez, A., Patiño, R. y Montes, D. (2012). La investigación formativa como una necesidad en el pre grado. Revista colombiana de ciencias, 4(1), pp. 1-3. Recuperado de: https://dialnet.unirioja.es/descarga/articulo/3968105.pdf

Guilar, M., (2009). Las idead de Brunner. "De la revolución cognitiva a la revolución cultural". Educere. 13(44), pp. 235-241. Recuperado de: https://www.redalyc.org/pdf/356/35614571028.pdf

Larraín, M. y González L. (25-26 de Julio 2005). Formación universitaria por Competencias. Seminario Internacional CINDA. Currículo universitario basado en competencias, p.44. Universidad del Norte, Barranquilla, Colombia. Recuperado de: https://n9.cl/3di6

Mendoza, H. (2016). Implementación del eje de investigación formativa en el plan curricular de la Facultad de Ingeniería de Minas de Universidad Nacional de Piura para la formación de profesionales investigadores - Piura 2015(Tesis Doctoral). Universidad Nacional de Piura, Perú. Recuperado de: http://repositorio.unp.edu.pe/handle/UNP/516

Moreno, T. (2011). Didáctica de la educación superior: Nuevos desafíos en el siglo XXI. Perspectiva educacional. 50(2), pp. 26-54. Recuperado de: https://n9.cl/oab58

Neidhardt, F. (1997). Michigan Tradition: Research and Undergraduate Education. Report to the Regents of the Universitay of Michigan. Recuperado de: www.research.umich.edu.

Ordoñez R. (2017). Aplicación del método heurístico y desarrollo de habilidades de investigación en estudiantes en la etapa de investigación formativa. Lima: UNMSM. Recuperado de: https://n9.cl/h74an

Restrepo, B. (2003). Investigación formativa e investigación productiva de conocimiento de la universidad. Nómadas, (18), pp. 195-202. Recuperado de: https://dialnet.unirioja.es/descarga/articulo/5137582.pdf

Rojas, J. (2018). Razonamiento cuantitativo y la investigación formativa en estudiantes de ingeniería de la Universidad Peruana de Ciencias Aplicadas, 2018 (Tesis 
Bryam Alejandro Colán-Hernández

Maestría). Lima: USMP. Recuperado de: http://www.repositorioacademico.usmp.edu.pe/handle/usmp/3922

Ruiz, A. (2017). Tesis Doctoral: Habilidades investigativas e investigación formativa con los estudiantes de la Universidad Nacional Autónoma de Chota. Lambayeque: UNPRG. Recuperado de: http://repositorio.unprg.edu.pe/handle/UNPRG/744

Ruiz, G. (2013). Teoría de la experiencia de John Dewey: Significación histórica y vigencia en el debate teórico contemporáneo. Foro de educación. 11(15), pp. 103124. Recuperado de: http://www.redalyc.org/pdf/4475/447544540006.pdf

Santaella, E. y Martinez, N., (2017). La pedagogía Freinet como alternativa al método tradicional de la enseñanza de las ciencias. Profesorado. Revista de curriculum y formación del profesorado. 21(4), pp. 359-379. Recuperado de: http://www.redalyc.org/pdf/567/56754639019.pdf

Santos, M. (2016). Investigación formativa en el aprendizaje del estudiante de enfermería en Culiacán, Sinaloa, México (Tesis Doctoral). Universidad Nacional de Trujillo, Perú. Recuperado de: http://dspace.unitru.edu.pe/handle/UNITRU/5899

Tunnerman, C., De Souza, M. (2003). Desafíos de la universidad en la sociedad del conocimiento, cinco años después de la conferencia mundial sobre educación superior. Forum ocasional papers series. (4). pp. 8. Recuperado de: https://n9.cl/tf65c 